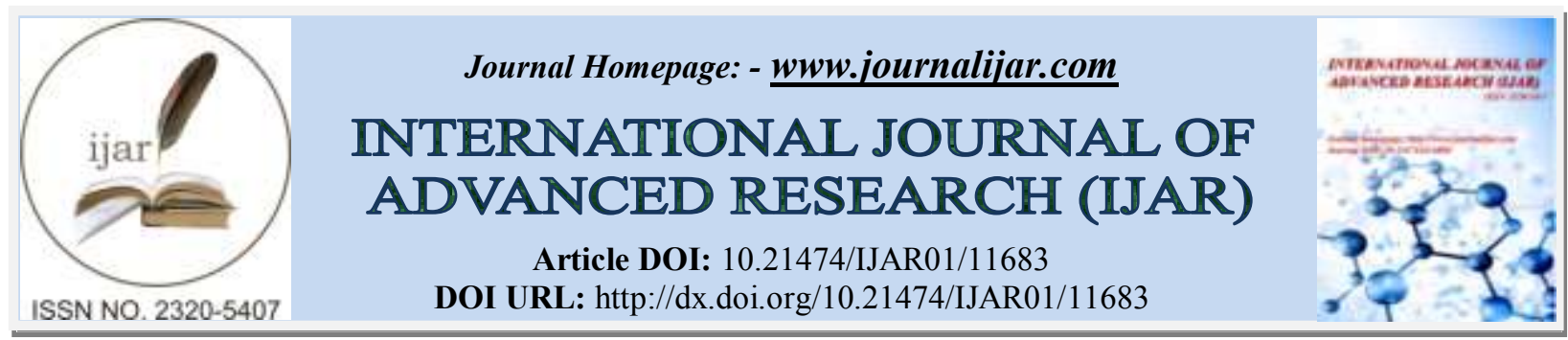

RESEARCH ARTICLE

\title{
EFFECTIVENESS OF FORWARD WALKING VERSUS BACKWARD WALKING ON CARDIOVASCULAR FITNESS IN OVERWEIGHT MIDDLE AGE PEOPLE
}

\author{
Dr. Heena Rathwa ${ }^{1}$, Dr. Advita Neville Deepak ${ }^{2}$ and Dr. Nikita Patel ${ }^{1}$ \\ 1. MPT Scholar, Parul Institute of Physiotherapy,Parul University, Vadodara. \\ 2. Associate Professor, Parul Institute of Physiotherapy, Parul University, Vadodara.
}

\section{Manuscript Info}

.........................

Manuscript History

Received: 10 July 2020

Final Accepted: 14 August 2020

Published: September 2020

Key words:-

Forward Walking, Backward Walking, 6

Minute Walk Test (6MWT), Rating

Perceived Exertion (RPE)

\section{Abstract}

Background:Overweightandobesityaredefinedasabnormalorexcessivef ataccumulationthatmayimpair health and also decrease endurance.

Methedology:SamplesofEighty $(n=80)$ indoorOverweightmiddleagedpe oplebetweentheaged of 40-60 years were included in the study. The subject who met the inclusion criteria was included in the study. An informed written consent from the subjects was taken. Subjects were divided into 40 in each group. The study is carried out for the duration of 6 months. Treatment was given 30 minutes for 6 days per week for 6 weeks. Outcome measures were taken on the 0 day and on the last day of the treatment with the use of 6 MINUTES WALK TEST.

Conclusion:Therefore we concluded that forward walking and backward walking both were effective but backward walking shows more effectiveness than forward walking in improving cardio-vascular fitness in overweight middle age people.

Copy Right, IJAR, 2020,. All rights reserved.

\section{Introduction:-}

Sedentary life style habits tend to be usually obese or overweight, from last few years physical therapy play important role in cardiovascular fitness by using different training like aerobics, anaerobic and walkingdifferent intervention are used to maintain healthy weight, muscle strength, joint mobility, strengthening the immune system and cardiovascular system. So just 30 minute every day walking improves cardio vascular fitness ${ }^{1}$. According to American heart association proved that more health risk in overweight and obese middle aged 40 to 60 years adults ${ }^{2}$. Many physiotherapy researches proved that walking is great way to improve maintain health ${ }^{3}$.

\section{Methodology:-}

This experimental study is to evaluate the effectiveness of forward walking versus backward walking on cardiovascular fitness in overweight middle age people. This study was conducted inParul Sevashram hospital,limda, Vadodara. Samples of Eighty $(n=80)$ indoor overweight middle aged people between the aged of 40 60 years were included in the study. The subject who met the inclusion criteria was included in the study. An informed written consent from the subject was taken. Initial cardio-respiratory assessment is done and subjects were divided into 40 in each group by the study is carried out for the duration of 6 months. The study was approved and conducted between 2018 and 2020 in accordance to the guidelines of the Parul University Institutional Ethics Committee for Human Research (PU - IECHR). Duration of treatment 30 minutes for 6 days per week for 6 
weeks.Outcome measures were taken on the 0 day and on the last day of the treatment with the use of 6 MINUTES WALKTEST.

\section{Inclusion Criteria:}

Both genders included, patient who is able to understand and follow simple verbal instruction, age between 40-60 years, patient who signed the inform consent form and Overweight people (BMI above25.0 to 29.9) (26kg/meter ${ }^{2}$ )

\section{Exclusion Criteria:}

History of cardiac and / or chronic pulmonary diseases with severe cardio pulmonary diseases, significant perceptual, cognitive, or communication impairments. Aphasia and history of thoracic and abdominal surgery, presence of a severe visual disability and visual field defects and the participants receiving any cardiopulmonary fitness training.

\section{Outcome Measure:}

6 minute walk test (6MWT) Before start assessing 6 minute walk test first of all we should check subjects HR (heart rate), SPO2 (oxygen saturation), BP (blood pressure), RPE (rating perceived exertion).

\section{Procedure}

Samples of 80 middle aged overweight people between the ages of $40-60$ years was taken for thestudy.Thesubjectswhomettheinclusioncriteriawereincludedinthestudy.Aninformed \& written consent was taken from the subjects. Initial assessment was done and subjects were divided in two groups, 40 subjects in each group by using chit method. Both the groups were receive 5 minutes warm up exercise at the beginning and 5 minutes cool down exercise at the end of the treatment period forrelaxation.

WARM-UP ACTIVITY-start slowly, do a few warm-up exercise (neck stretch, biceps stretch forearm(flexion. extension ) stretch, pectoralis stretch, back side stretch, wrist roll, elbow flexion, shoulder girdle, ankle toe, side to side step) and General stretches first. In COOL DOWN -ask subject to walk for 5 minutes at lower pace GROUP A: FORWARD WALKING GROUP B: BACKWARD WALKING GROUP A: FORWARD WALKING

Total treatment time: 30 minutes (5 minutes warm up, 20 minutes forward walking, 5 minutes cool down)

Before starting forward walking all the subject was examined by six minute walk test. After doing warm up exercises ask the subject was asked to start forward brisk walking and instructed to continue for 20 minutes. Instruct the subject that stop walking whenever feels fatigue or any other problem and take rest.

\section{Group B: Backward Walking}

Total treatment time: 30 minutes (5 minutes warm up, 20 minutes backward walking, 5 minutes cool down)

\section{Backward Walking-}

Backward walking at the dryland first demonstrated by the therapist1.AfterdoingwarmupexercisesaskthesubjectwasaskedtostartBackwardwalking and instruct to continue for 20 minutes.

Instructed the subject that stop walking whenever he feels fatigue or any other problem and take rest.

\section{Statistical analysis \\ Statistical methods}

Descriptive statistical analysis was carried out in the present study. Outcome measurements were measured using 6 MWT presented as mean $\pm \mathrm{SD}$. significance was assessed at $5 \%$ levelof significance $\mathrm{p}<0.005$ ( 2 -tailed hypothesis test considered).

\section{Statistical tests}

Paired' $t$ 'test as a parametric had been used for analysis of 6 MWT within the group A andgroup B with calculation of percentage of change. 
Independent' $t$ ' test as a parametric had been used the compare the means of 6 MWT between the groups with calculation of percentage of difference between the means.

\section{Statistical software:}

The statistical software namely SPSS 2.1.0.1 will be used for the analysis of the data and Microsoft word and excel had been used to generate graphs, tables etc.

Represent The Ratio Of Male And Female In Group A And Group B
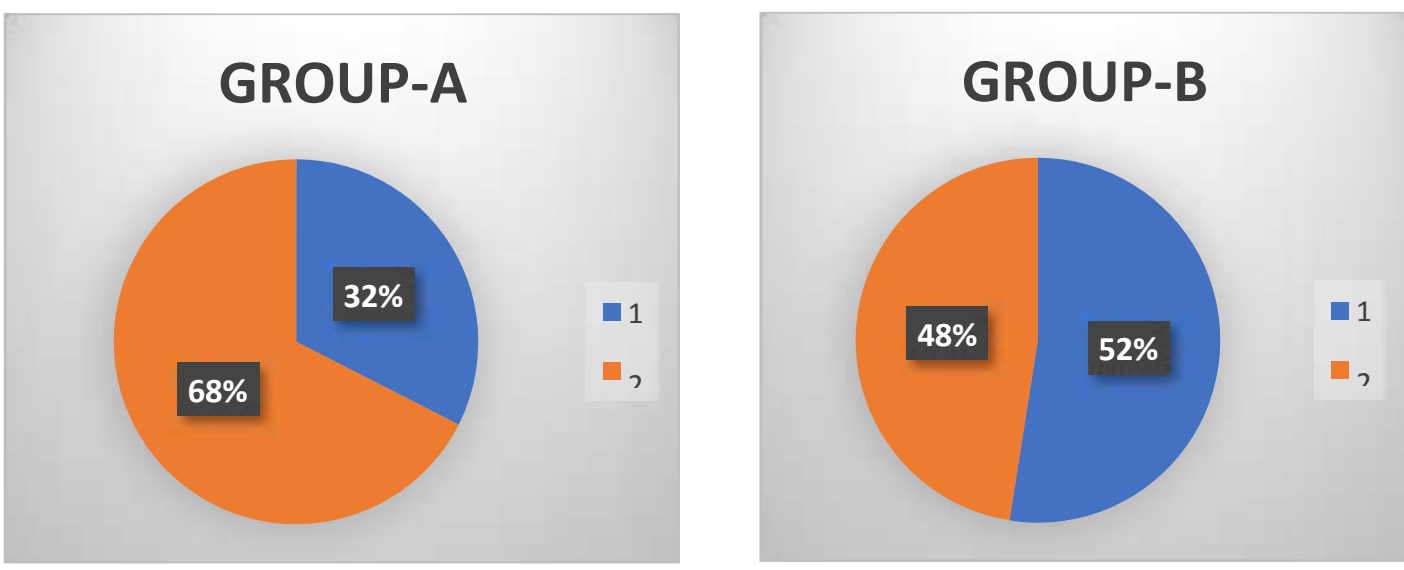

Represent Intra Group Comparision For 6 Mwt Group-A

\begin{tabular}{|l|l|l|l|l|l|}
\hline \multirow{2}{*}{ GROUP } & \multirow{2}{*}{ OUTCOME } & PRE & POST & $\begin{array}{l}\text { T- } \\
\text { VALUE }\end{array}$ & $\begin{array}{l}\text { P- } \\
\text { VALUE }\end{array}$ \\
\cline { 3 - 5 } & & MEAN/SD & MEAN/SD & & \\
\hline $\begin{array}{l}\text { GROUP- } \\
\text { A }\end{array}$ & 6MWT & $\begin{array}{l}\mathbf{1 8 1 . 0 0 0 0} \\
\pm 12.56777\end{array}$ & $\mathbf{1 9 2 . 7 5 0 0} \pm 14.13987$ & $\mathbf{- 9 . 9 4 5}$ & $\mathbf{0 . 0 0 1}$ \\
\hline
\end{tabular}

\section{Intragroup analysis:}

Theabovetableshowstheintragroupcomparisonof6MWTscorefor

functionalassessmentinGroupA.Thecomparisonwasdonethroughpairedttest.Thepvalue of Group A comparing pre and post treatment score of $6 \mathrm{MWT}$ is 0.001 . The $\mathrm{p}$ value is $<0.001$ which shows that Group A significant in improving 6MWTscore.

So, Null hypothesis is $\mathrm{H} 0$ is rejected and alternate hypothesis $\mathrm{H} 1$ is accepted.

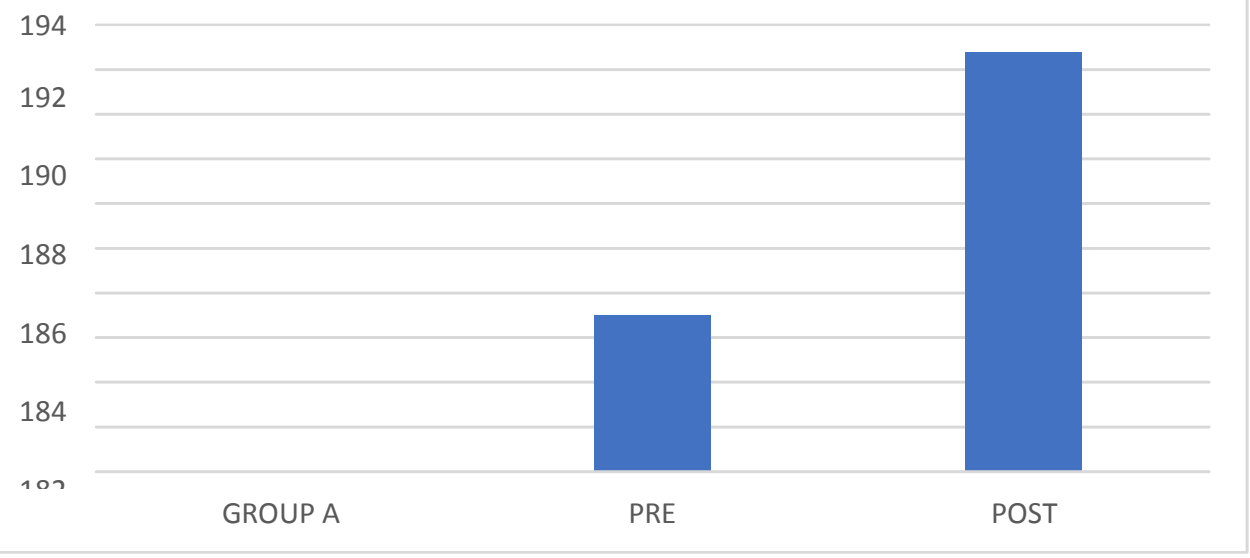


1) 6MWT: PRE POST COMPARISON (GROUP A)

represent inter group comparision for 6 mwt group-B

\begin{tabular}{|l|l|l|l|l|l|}
\hline GROUP & \multirow{2}{*}{ OUTCOME } & PRE & POST & \multirow{2}{*}{ T-VALUE } & \multirow{2}{*}{ P-VALUE } \\
\cline { 3 - 4 } & & MEAN/SD & MEAN/SD & & \\
\hline GROUP-B & 6MWT & $\mathbf{1 8 1 . 0 0 0 0}$ & $\mathbf{1 9 1 . 5 0 0 0 0}$ & $\mathbf{- 1 7 . 0 4 6}$ & $\mathbf{0 . 0 0 1}$ \\
& & \pm 8.77058 & \pm 9.21259 & & \\
\hline
\end{tabular}

Inter group analysis:

The above table shows the intra group comparison of 6 MWT scorefor functional assessment in Group B. the comparison was done through paired t test. The $p$ value of Group A comparing pre and post treatment score of 6MWT is 0.001 . The $\mathrm{p}$ value is $<0.001$ which Group B significant in improving 6MWTscores.

So, Null hypothesis is $\mathrm{H} 0$ is rejected and alternate hypothesis $\mathrm{H} 1$ is accepted.

6 MWT: PRE POST COMPARISON (GROUP B)

Represents Inter Group Comparison For 6MWT

\begin{tabular}{|l|l|l|c|c|}
\hline & GROUP & MEAN/SD & T-VELUE & P-VELUE \\
\hline 6 MWT & GROUP-A & $-12.000 \pm$ & & \\
& & 6.07644 & -426 & 0.671 \\
& GROUP-B & -11.5000 & & \\
& & \pm 4.26675 & & \\
\hline
\end{tabular}

Theabovetableshowsinter-groupcomparisonofposttreatment6MWTscoresforfunctional

assessmentofgroupAandB.theanalysiswascarriedoutby.Atbaseline,

the pvalueis $<0.001$.Itshowsthatthereissignificantdifferencebetweentheposts-treatmentsscoresofboth

groups. ThevaluecomparingtheposttreatmentscoreforGroupAandGroupBis0.671which is suggestive of significant improvement bothgroups.

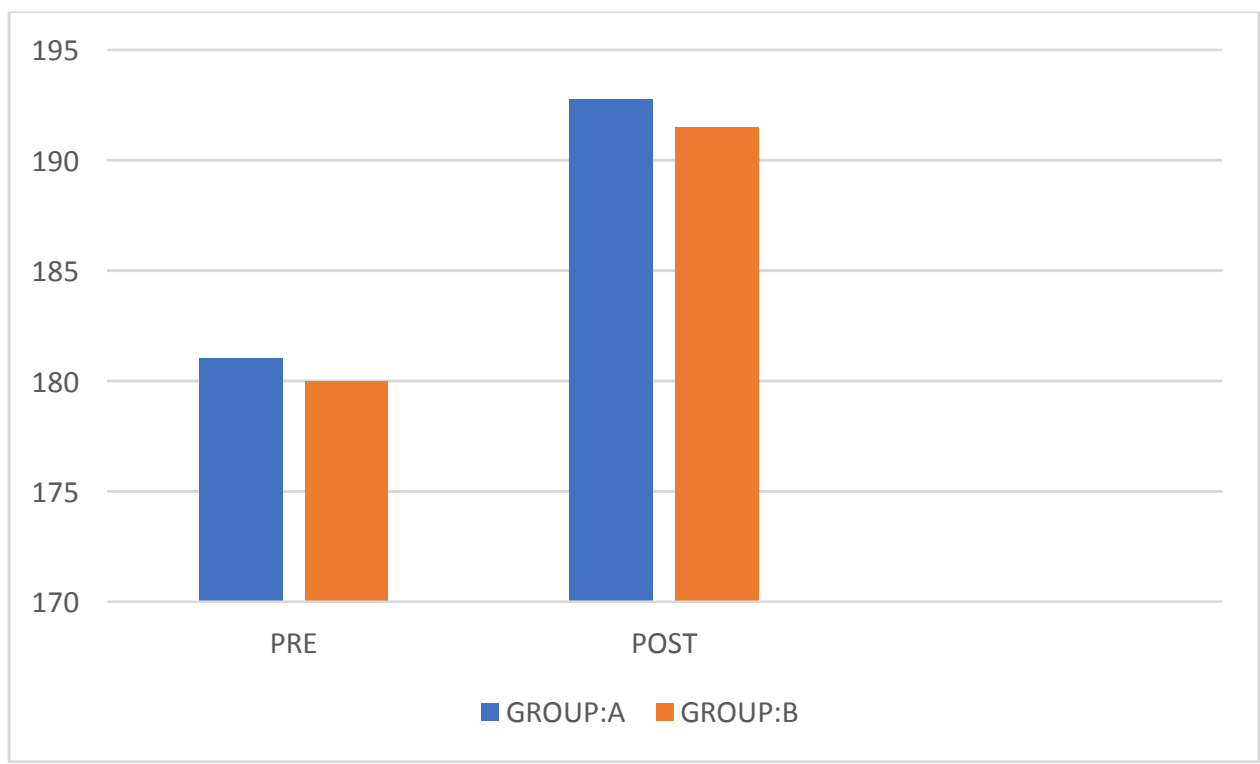

\section{Discussion:-}

Cardio-vascularfitnessreducesinoverweightmiddlepeople, soourstudywasfocusonhowto increase cardio-vascular fitness in overweight people. For that my treatment protocol was forward walking and backward walking in overweight middle age people, so it helps in improving cardio-vascularfitness. 
Statistically, intra group analysis: the comparison of pre and post treatment scores for cardio- vascular fitness shows that $\mathrm{P}$-value is $<0.001$ for 6 minute walk test (6MWT) in both groups.

Statistically, intergroupanalysis:thecomparisonofposttreatmentscoreforcardio -vascular fitness shows that P-value is $<0.001$ for 6 minute walk test (6MWT). Group-B backward walking more significant rather than Group-A forward walking according to mean of6MWT.

Overweightisandincreasingbodyweightasaresultexcessaccumulationoffatandisaresult when calorie value of food intake exceeds energy output. The prevalence of overweight increasing and is associated with numerous chronic conditions therefore it is important to implement effective interventions for treatment of excess body weight.

Walking is a rhythmic, dynamic, aerobic activity of large skeletal muscles that confers the multifarious benefits with minimal adverseeffects.

Forward walking is probably one of the most common modes of aerobic conditioning and numerous studies have shows the benefits of different kind's forward walking training

Backward walking is a training technique often used in team sports to increase co- ordination and endurance, however, the effect of backward walking training program on the aerobic fitness of healthy individuals has not been qualified before. The results of this study provideforthefirsttime,evidencethatbackwardwalkingcanimprovecardiovascularfitness in overweight middle agepeople.

In the order to develop and maintain cardio-vascular fitness it is recommended that both men and women exercise of intensity that correspond to $65-90 \%$ of maximal heart rate or $50-85 \%$ of Vo2max. The most important and novel finding of this study was that the people following the 6 week training significantly improve cardio-vascular fitness.

Althoughithasbeensuggestedthatbackwardwalkingtrainingincreasequadricepspowerand strength .similar effects were not found in thisstudy.

Mackie et al, (2018) rained their subject for three months. On a treadmill and showed that the power of the subjects legs increased significantly, while the strength of the knee flexors and extensors decreased in most subjects.

Themechanismforthatimprovementinquadricepsstrengthisprobablyrelatedtotheisometric and concentric nature of the muscle actions during backward walking, as well as that fact that the leg muscles are active for a longer sustained period during backward walking comparedto forward walking.

In our current study we used and exercise group that initiated the treatment which prescribed for 6 days for week for 6 week.

As the results outcome measures 6 MWT the Group-A forward walking as well as Group-B backwardwalkingindicatedbetterimprovementincardio-vascularfitnessbutoninter-group comparison more improvement seen in Group $-\mathrm{B}$ as compared to Group-A we therefore speculatethatpossiblereasonforthiscouldbeGroup-Bbackwardwalkingresultsinincreased metabolic cost and cardiovasculardemand.

The increased metabolic cost during backward locomotion is due to novel activity which requires increasingly greater motor unit recruitment in order to complete the task. Backward walking consumes more energy and thus helps in improving aerobic capacity and cardio vascular fitness So, Group-B helps increased cardio-vascular fitness more than Group-A.

According to hypothesis it is proven that backward walking is more effective in increasing cardio-vascular fitness in overweight middle age people.

Conclusion: This research demonstrated that backward walking showing improvement in cardiovascular fitness. These findings indicate that backward walking could be used to improving cardio vascular fitness with overweight middle age people.

\section{Conflict of Interest:}

None 


\section{Source of Funding:}

Self

\section{Ethical Clearance:}

Parul University Institutional Ethics Committee for Human Research (PU - IECHR)

\section{Refrences:-}

1. Saksvig BI, Catellier DJ, Pfeiffer K, Schmitz KH, Conway T, Going S, Ward D, Strikmiller P, Treuth MS. Travel by walking before and after school and physical activity among adolescent girls. Archives of pediatrics \& adolescent medicine. 2007 Feb 1;161(2):153-8.

2. Steinberger J, Daniels SR. Obesity, insulin resistance, diabetes, and cardiovascular risk in children: an American Heart Association scientific statement from the Atherosclerosis, Hypertension, and Obesity in the Young Committee (Council on Cardiovascular Disease in the Young) and the Diabetes Committee (Council on Nutrition, Physical Activity, and Metabolism). Circulation. 2003 Mar 18;107(10):1448-53.

3. Demers C, McKelvie RS, Negassa A, Yusuf S, RESOLVD Pilot StudyInvestigators. Reliability, validity, and responsiveness of the six-minutewalk test in patients with heart failure. American heart journal. 2001 Oct 1;142(4):698-703.

4. Dr Mike Brannan M, Varney J, Timpson C.10 minutes brisk walking each day in mid-life for health benefits and achievement of recommended activity levels - evidence summary. 20170ct;29:4.

5. Boston, ma, lancet, Yuan Lu, Cut heart disease, stroke risks by more than half.[online] Harvard Global Health Institute, 15july2013.617.432.8413.

6. Better health channel, state of Victoria, walking good health, Heart Foundation walkinggroup,2019.13:11:12,

7. Sipila SA, Suominen HA. Effects of strength and endurance training on thigh and legmusclemassandcompositioninelderlywomen.Journalofappliedphysiology. 1995 Jan 1;78(1):334-40.

8. Cadore EL, Pinto RS, Bottaro M, Izquierdo M. Strength and endurance training prescription in healthy and frail elderly. Aging and disease. 2014Jun;5(3):183.

9. LauferY.Effectofageoncharacteristicsofforwardandbackwardgaitatpreferred

andacceleratedwalkingspeedA:ThejournalofGerontologySeriesA:Biological Sciences and Medical Sciences. 2005 May1;60(5):627-32.

10. Baker G, Grey SR, Wright A, Fitzsimons C, Nimmo M, Lowry R, Mutrie N, Scottish Physical Activity Research Collaboration. The effect of a pedometer - based Community walking intervention "Walking for Wellbeing in the West" on physicalactivitylevelsandhealthoutcomes:a12weekrandomizedcontrolledtrial. International journal of Behavioural Nutrition and physical Activity .2008 Dec 1;(1):44. 